\title{
Sum Frequency Generation Vibrational Spectroscopy Characterization of Surface Monolayers: Catalytic Reaction Intermediates and Polymer Surfaces *
}

\author{
Gabor A. Somorjai ${ }^{\dagger}$ Keng C. Chou, and Minchul Yang \\ Department of Chemistry and Lawrence Berkeley National Laboratory University of California, Berkeley, CA 94720
}

(Received 4 November 2003; Accepted 10 February 2004; Published 28 February 2004)

\begin{abstract}
Sum frequency generation (SFG) vibrational spectroscopy is highly surface sensitive because the second-order nonlinear optical process is only allowed in media without inversion symmetry, such as interfaces. As an all-optical technique, SFG can be employed to study molecular compositions and structures at solid/liquid and at solid/high pressure gas interfaces. This paper discusses the applications of SFG in our laboratory to study the reaction intermediates of catalytic reactions on metal surfaces under high-pressure conditions, the surface composition and structure of polymers, and the interfacial properties of proteins on liquid/solid interfaces. [DOI: 10.1380/ejssnt.2004.106]
\end{abstract}

Keywords: Sum frequency generation; Nanoparticle; Catalysis; Surface chemical reaction; Polymer; Surface segregation

\section{INTRODUCTION}

Studies of surfaces on the atomic and molecular level under vacuum or low-pressure conditions are well served by electron scattering, ion scattering, and molecular beam scattering techniques. However, solid/high-pressure gas and solid/liquid interfaces could not be studied by the same techniques because the probing beams suffer from intensive scattering before they reach the surface. In recent years, photon-in photon-out techniques as well as scanning probes, such as scanning tunneling and atomic force microscopies, have provided the means to study these buried interfaces. The purpose of this paper is to demonstrate sum frequency generation (SFG) surface vibrational spectroscopy as a powerful technique for the studies of the solid/high-pressure gas and solid/liquid interfaces. The nonlinear spectroscopic technique has its origin in second harmonic generation that was first demonstrated by Franken in 1961 [1] and formulated by Bloembergen who received the Nobel Prize for it in 1981. In Berkeley, Professor Yuen-Ron Shen and myself have developed SFG vibrational spectroscopy by tuning one of the laser beams in the infrared region and keeping the
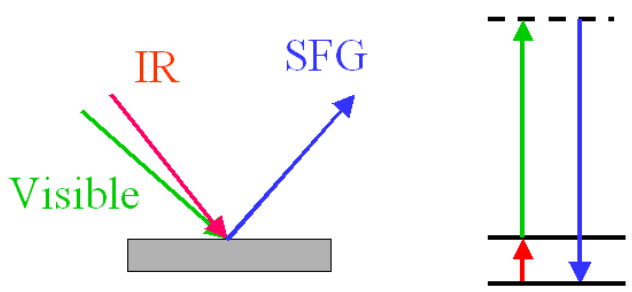

FIG. 1: The scheme of IR-visible SFG experiments. The process is a combination of a vibrational excitation and an antiStoke Raman transition.

*This paper was presented at The 4th International Symposium on Atomic Level Characterizations for New Materials and Devices (ALC '03), Kauai, Hawaii, USA, 5-10 October, 2003.

†Corresponding author: somorjai@socrates.berkeley.edu
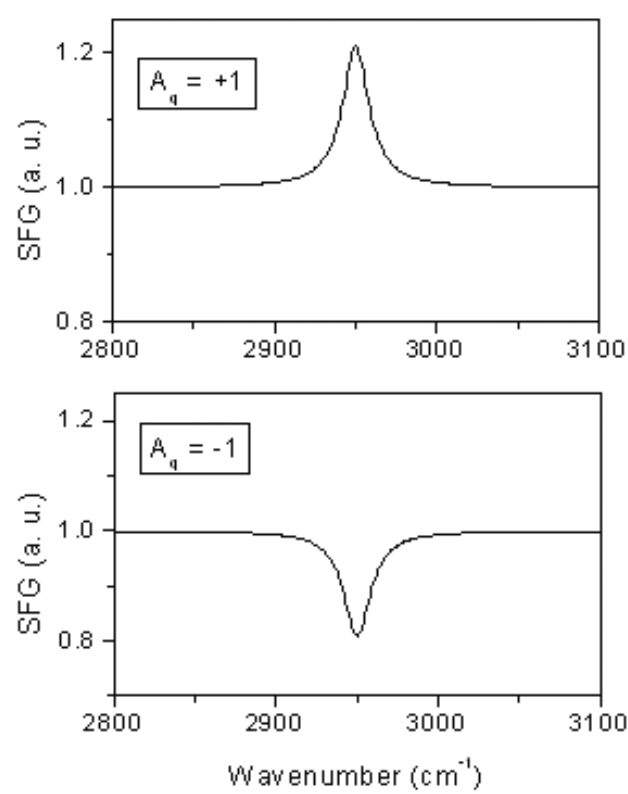

FIG. 2: Calculated SFG spectra lineshapes using Eq. (1) with $\chi_{N R}^{(2)}=1, \phi=-90^{\circ}, \omega_{q}=2950 \mathrm{~cm}^{-1}, \Gamma_{q}=10 \mathrm{~cm}^{-1}$, and $A_{q}=1$ and -1 , respectively.

other laser beam in the visible range, providing an SFG vibrational spectrum in the visible range. It is a highly species- and surface-specific technique to study molecular structures and chemical compositions at interfaces. This paper discusses the applications of SFG to study catalytic reactions under high pressure and electrochemical conditions, the surface structures and segregations of polymers, and protein adsorption at liquid/solid interfaces.

\section{EXPERIMENTAI}

Figure 1 shows the scheme diagrams of SFG experiments. They were carried out by mixing a visible beam (frequency $\omega_{v i s}$ ) and a frequency-tunable IR beam (frequency $\left.\omega_{i r}\right)$ on the surfaces to generate a third beam with a frequency $\omega_{S F G}=\omega_{\text {vis }}+\omega_{i r}$. The SFG from the surface is enhanced, as the IR frequency is resonant with the vibrational frequency of surface molecules [2]. The inten- 


\section{Top View of Reaction Cell}

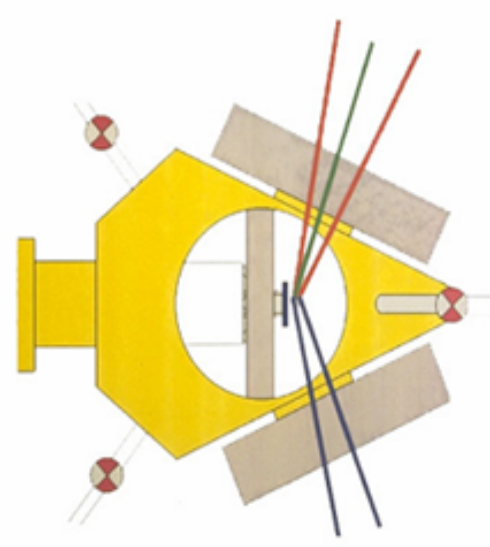

\section{Electrochemical Cell}

$560 \mathrm{ml}$ in Volume

$2 \mathrm{Ca} \mathrm{F}_{2}$ Windows

GC Line and

Re-circulation Pump

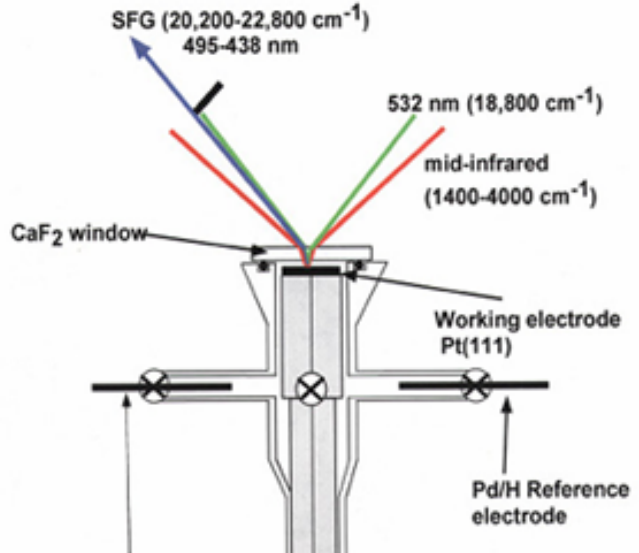

\section{Polymer/vapor and Polymer/liquid Interfaces}
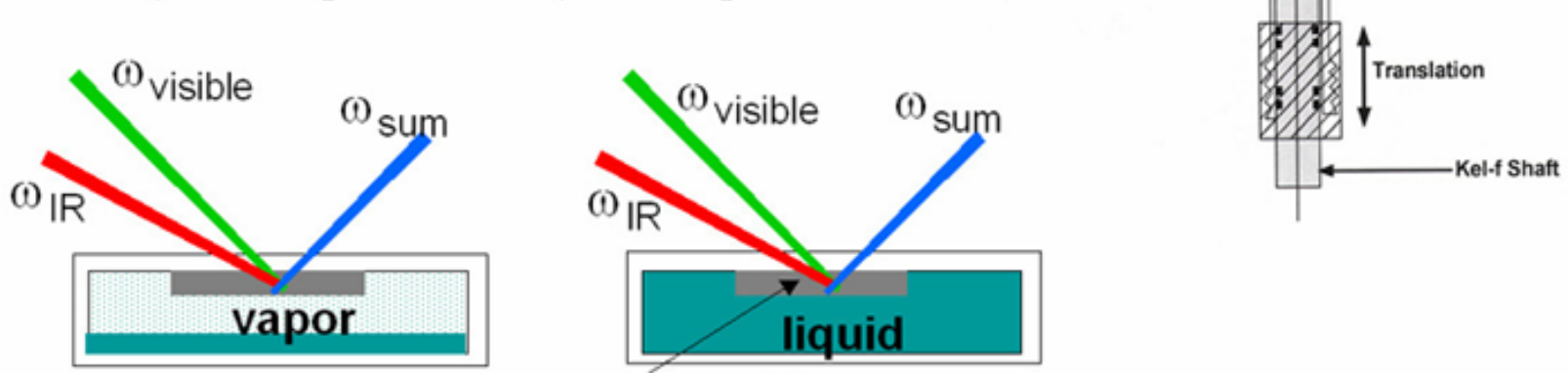

polymer film

FIG. 3: The experimental configurations for the study of solid/high-pressure gas and solid/liquid interfaces using SFG.

sity of the reflected SFG is proportional to the square of the surface second order nonlinear susceptibility:

$$
\begin{aligned}
I_{S F G} & \propto\left|\chi_{N R}^{(2)}+\chi_{R}^{(2)}\right|^{2} \\
& =|| \chi_{N R}^{(2)}\left|e^{i \phi}+\sum_{q} \frac{A_{q}}{\omega_{I R}-\omega_{q}+i \Gamma_{q}}\right|^{2}
\end{aligned}
$$

with

$$
\begin{aligned}
A_{q, i j k} & =N_{s} \sum_{l m n} a_{q, l m n}\langle(\mathbf{i} \cdot \mathbf{l})(\mathbf{j} \cdot \mathbf{m})(\mathbf{k} \cdot \mathbf{n})\rangle \\
a_{q, l m n} & =-\frac{1}{2 \epsilon_{0} \omega_{q}} \frac{\partial \mu_{n}}{\partial Q_{q}} \frac{\partial \alpha_{l m}}{\partial Q_{q}}
\end{aligned}
$$

where $\chi_{N R}^{(2)}, \chi_{R}^{(2)}, \phi, A_{q}, \omega_{q}, \Gamma,<>, \partial \mu_{n} / \partial Q_{q}$, and $\partial \alpha_{l m} / \partial Q_{q}$ are the non-resonant second order susceptibility, resonant second order susceptibility, phase, amplitude, resonant frequency of $q$-th vibrational mode, width, orientation average, infrared dipole derivative, and Raman polarizability tensor, respectively. SFG is a combination of a vibrational excitation and an anti-Stoke Raman transition; therefore both IR and Raman selection rules must be obeyed. SFG can happen only in media that has no inversion symmetry or non-centro symmetric, such as interfaces [3]. Many of condensed phases are centro-symmetric, and gas phases are isotropic, yielding no SFG signal. In contrast, the inversion symmetry is broken at interfaces; therefore, SFG is allowed at interfaces. As a consequence, SFG is a highly species- and surface-sensitive technique with sub-monolayer sensitivity. The lineshape of an SFG spectrum is sensitive to the orientation average of surface molecules, as shown in Eq. (2). Figure 2 shows examples of how the orientation average can change the lineshape of an SFG spectrum. Peaks in an SFG spectrum can be positive-going or negativegoing, depending on the relative phase of $\chi_{N R}^{(2)}$ and $\chi_{R}^{(2)}$. Examples will be discussed in the following section.

Figure 3 shows a few experimental configurations to study the solid/high-pressure gas and solid/liquid interfaces using SFG, including a high-pressure catalytic reaction chamber [4], an electrochemical cell with a single crystal electrode [5], and a polymer vapor or polymer liquid cell [6] where the probing beams are introduced through the window of the cell.

\section{RESULTS AND DISCUSSION}

\section{A. Surface Sensitivity of SFG}

To demonstrate the surface sensitivity of SFG, Fig. 4 shows that the SFG intensity from a poly-(ethylene gly- 
FTIR Spectra

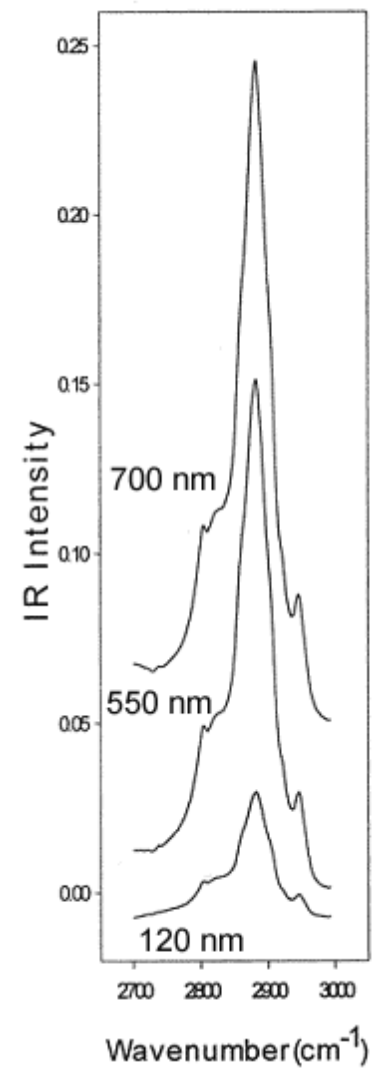

SFG Spectra

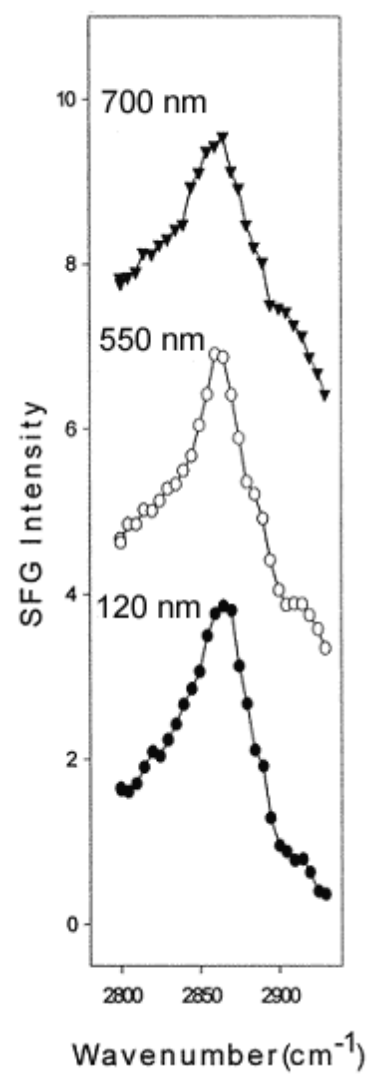

FIG. 4: The IR absorption spectra and SFG Spectra of poly-(ethylene glycol) samples with different thickness.
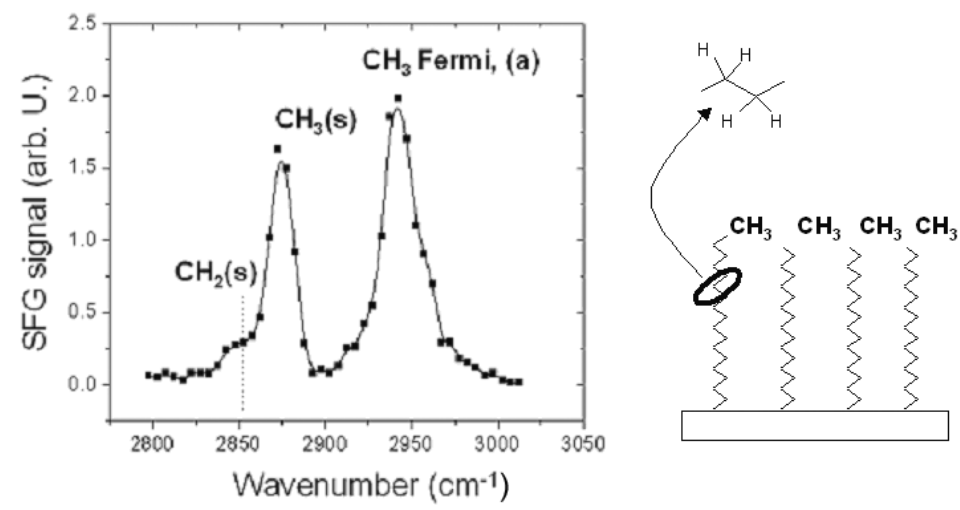

FIG. 5: The SFG spectrum from a self-assembled monolayer.

col) film remains constant when the film thickness increases [7]. In comparison, the intensity of the infrared absorption spectrum increases in proportion to the film thickness. Thus this clearly shows that SFG is a surface sensitive technique while infrared absorption spectroscopy is dominated by bulk signals. As another example, Fig. 5 shows the SFG spectrum from a self-assembled monolayer. The $\mathrm{CH}_{2}$ groups, which are in all-trans-configuration, give no SFG vibrational spectrum because they have inversion symmetry. Only the $\mathrm{CH} 3$ end groups appear in the SFG spectrum.

The orientation of molecules can also be obtained from the SFG spectrum since the amplitude, $A_{q, i j k}$, depends on their orientational average, as described in Eq. (2). This effect is clearly seen in an electrochemical experiment where the orientation of molecules can be manipulated by an external potential. Figure 6 shows the SFG spectra of acetonitrile on a $\mathrm{Pt}(111)$ electrode at two different potentials. As the applied potential increases, the molecule flips around from a structure with the $\mathrm{CH}_{3}$ group directing toward the surface to a structure with the CN group directing toward the surface [8]. 

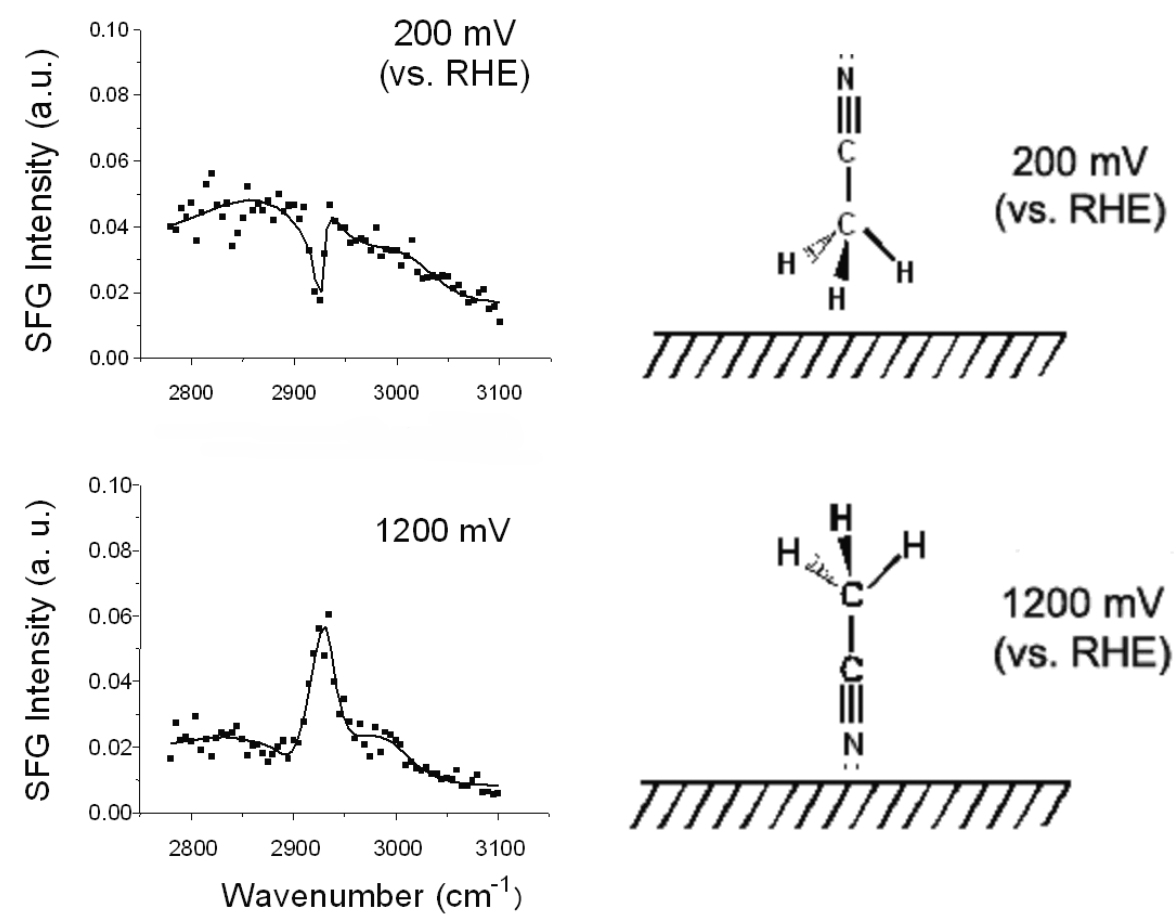

FIG. 6: SFG spectra of 0.05 mole fraction $\mathrm{H}_{2} \mathrm{O}+$ acetonitrile solution on a $\mathrm{Pt}(111)$ electrode at potential 200 and $1200 \mathrm{mV}$ (vs. RHE), respectively.

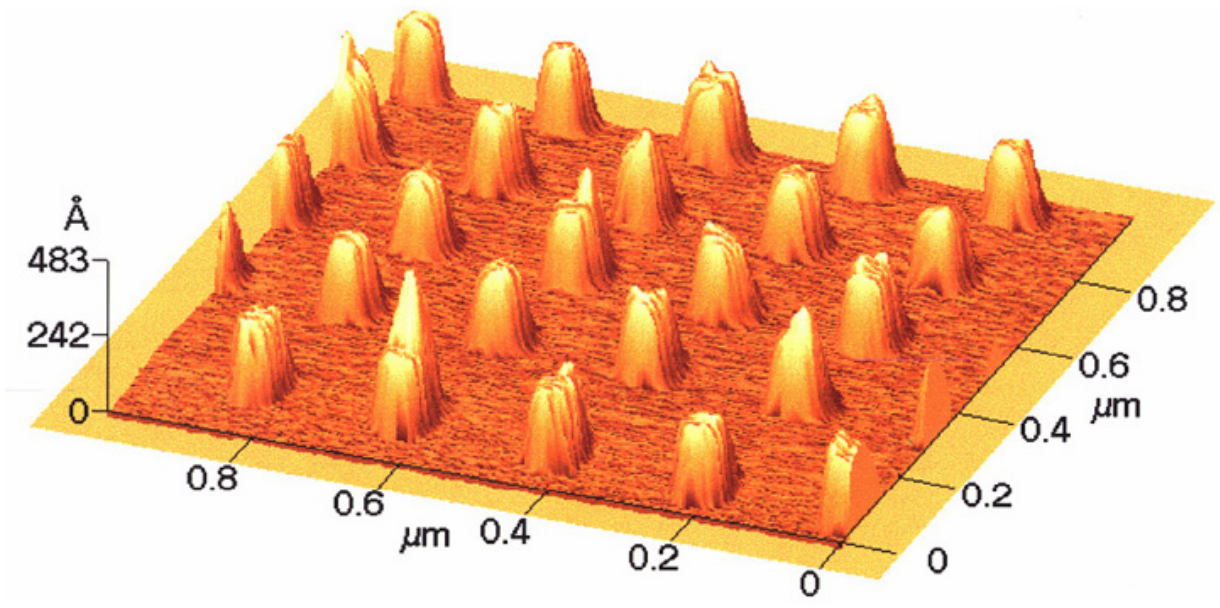

FIG. 7: The AFM image of platinum nanoparticle array with diameter of $50 \mathrm{~nm}$ and interparticle spacing of $200 \mathrm{~nm}$.

B. SFG Signals from Nanoparticles

Using electron beam lithography one can fabricate an ordered array of platinum nanoparticles, as shown in Fig. 7. When carbon monoxide adsorbs on the nanoparticles with a particle size of around $50 \mathrm{~nm}$, a more than 10,000 fold increase in the SFG intensity of CO molecules has been observed, as shown in Fig. 8 [9]. This is due to the plasmon resonance in small metal particles. SFG provides a method for studying model nanoparticle catalysts. Without the surface enhancement, the vibrational spectra of adsorbates at low coverage, $<5 \%$ of a monolayer in this case, would be unattainable. The enhanced signal allows the study of catalytic reactions on model nanopar- ticle surfaces.

\section{High-pressure Surface Science}

The study of surface science under high-pressure conditions has been difficult, mostly because the lack of probing tools. Although IR absorption spectroscopy has been widely used to study surface reactions under low-pressure conditions, it has suffered from the strong absorption of IR by the background gases under high-pressure conditions. Second-harmonic generation (SHG) is surface sensitive even under high-pressure conditions, but it is not species-sensitive. The development of IR-visible sum fre- 


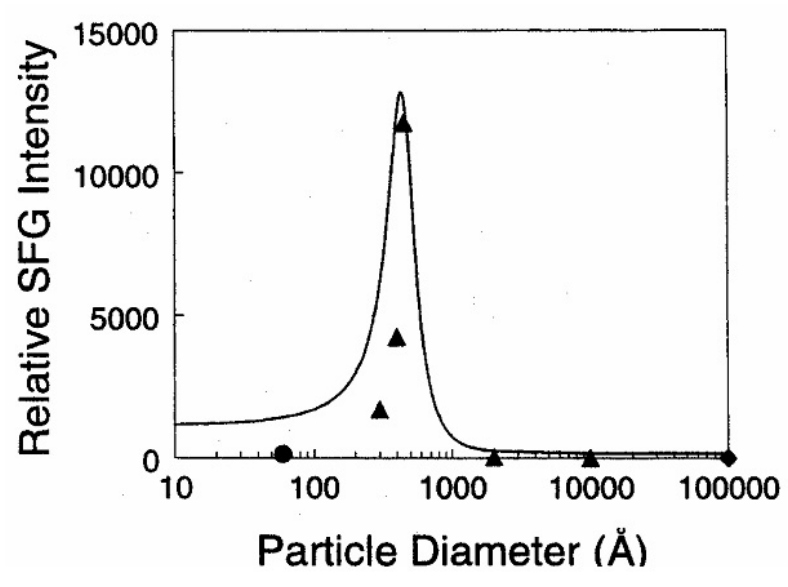

FIG. 8: The enhancement of SFG signals from CO adsorbed on $\mathrm{Pt}$ nanoparticles as a function of particle size. The triangles represent the nanoparticle arrays fabricated with e-beam lithography. The circle represents Pt clusters deposited by evaporation. The diamond represents $\mathrm{Pt}$ metal film. Solid line is the predicted intensity based on plasmon resonance and substrate contributions to the local fields.

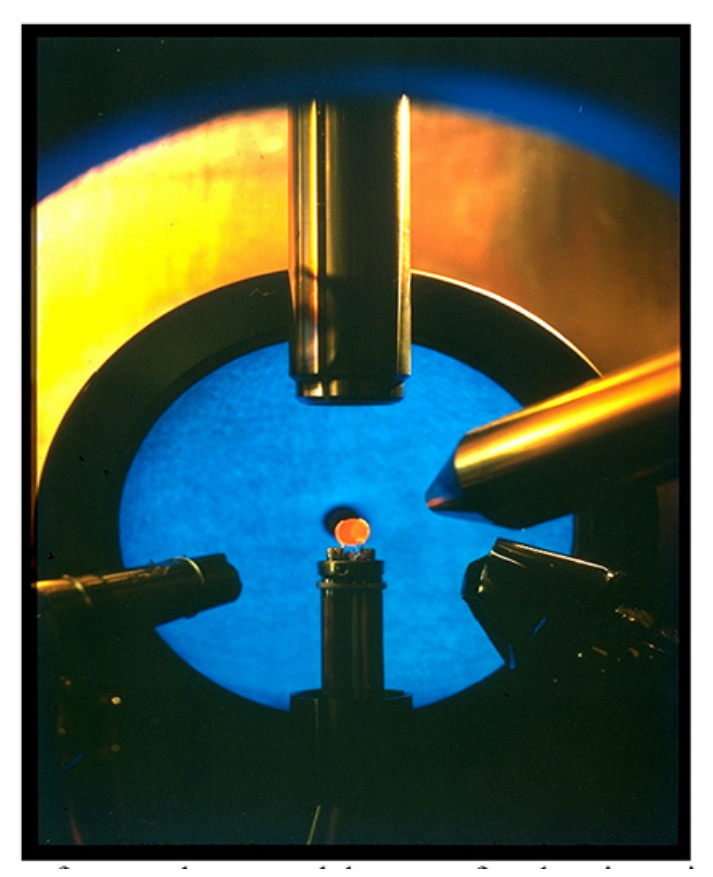

FIG. 9: The single crystal surface used as a model system for chemisorption and catalytic reaction studies.

quency generation SFG has helped bridge the pressure gap between studies of heterogeneous catalysis under high vacuum and atmospheric pressure. Due to SFGfs surface sensitivity, SFG can only be generated from the surface. Therefore, a background intensity subtraction is not needed. By in situ monitoring the SFG vibrational spectra of surface species at high pressure, one can correlate the results with the simultaneously measured reaction rate using gas chromatography.

Figure 9 shows a single crystal surface that is used as a model catalyst for chemisorption and catalytic reaction studies. As shown in Fig. 10, SFG allows measurements
(A)

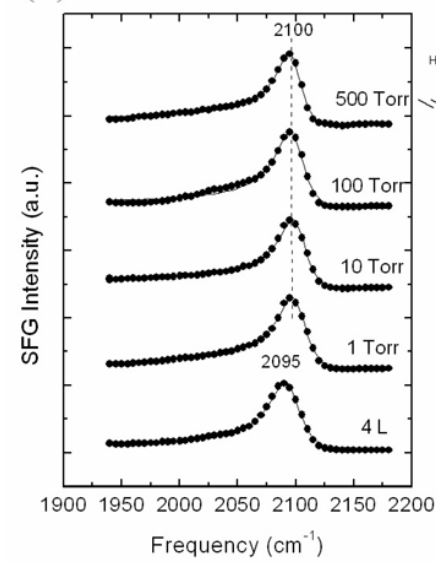

(B)

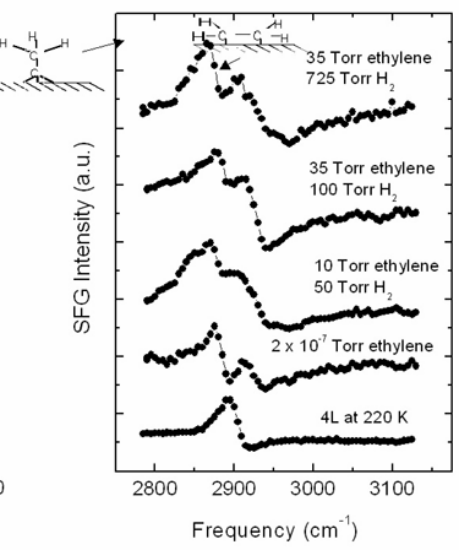

FIG. 10: The SFG spectra of (A) CO on $\mathrm{Pt}(557)$ and (B) ethylene on $\mathrm{Pt}(100)$.
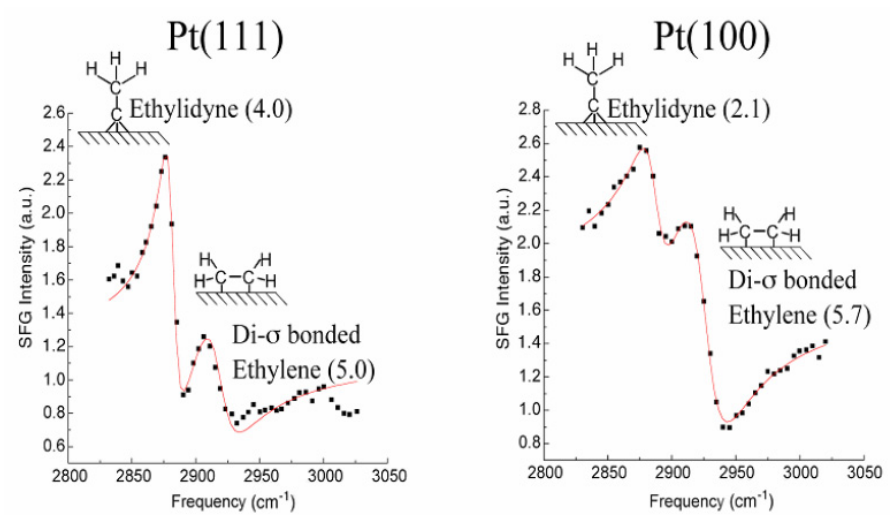

FIG. 11: The SFG vibrational spectra obtained during ethylene hydrogenation to ethane on the $\mathrm{Pt}(111)$ and $\mathrm{Pt}(100)$ surfaces at $300 \mathrm{~K}$.

of the vibrational spectrum of carbon monoxide or ethylene respectively over 10 orders of magnitude pressure range without significant reduction of spectral intensities. Here, we use ethylene hydrogenation reactions to show how one can study catalytic reactions by in-situ measurements of SFG vibrational spectra. Figure 11 shows the SFG vibrational spectra obtained during ethylene hydrogenation to ethane on the $\mathrm{Pt}(111)$ and $\mathrm{Pt}(100)$ surfaces at $300 \mathrm{~K}$ [10]. The turnover rate for the reaction (how many ethane molecules are produced per platinum site per second) is identical on the two crystal faces. The surface vibrational spectra show the presence of both ethylidyne and di- $\sigma$ bonded ethylene but with different coverage. At higher pressures one can also see two additional species in the SFG spectra, $\mathrm{C}_{2} \mathrm{H}_{5}$ and $\pi$-bonded ethylene. These species are those that turn over during the reaction because they are weakly bound. They readily desorb from the surface when the system is depressurized. The ease of adsorption and desorption, which is due to their weak bonding to the surface, makes them reactive surface intermediates. Ethylidyne and di- $\sigma$-ethylene, which are also present on the surface when the system is evacuated or reduced to low pressures, are strongly bound and therefore 


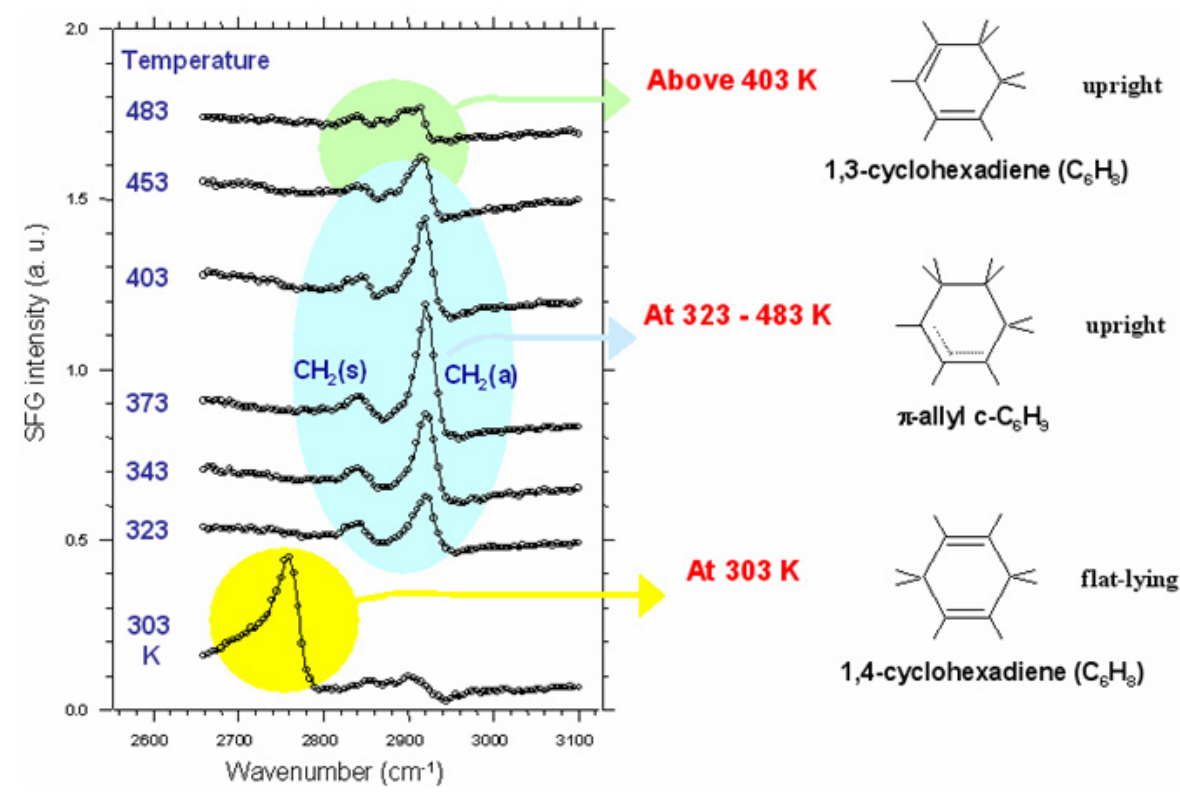

FIG. 12: Temperature-dependent SFG spectra and corresponding surface species on Pt(111) under 1.5 Torr cyclohexene and 15 Torr $\mathrm{H}_{2}$ in the temperature range $303-483 \mathrm{~K}$.

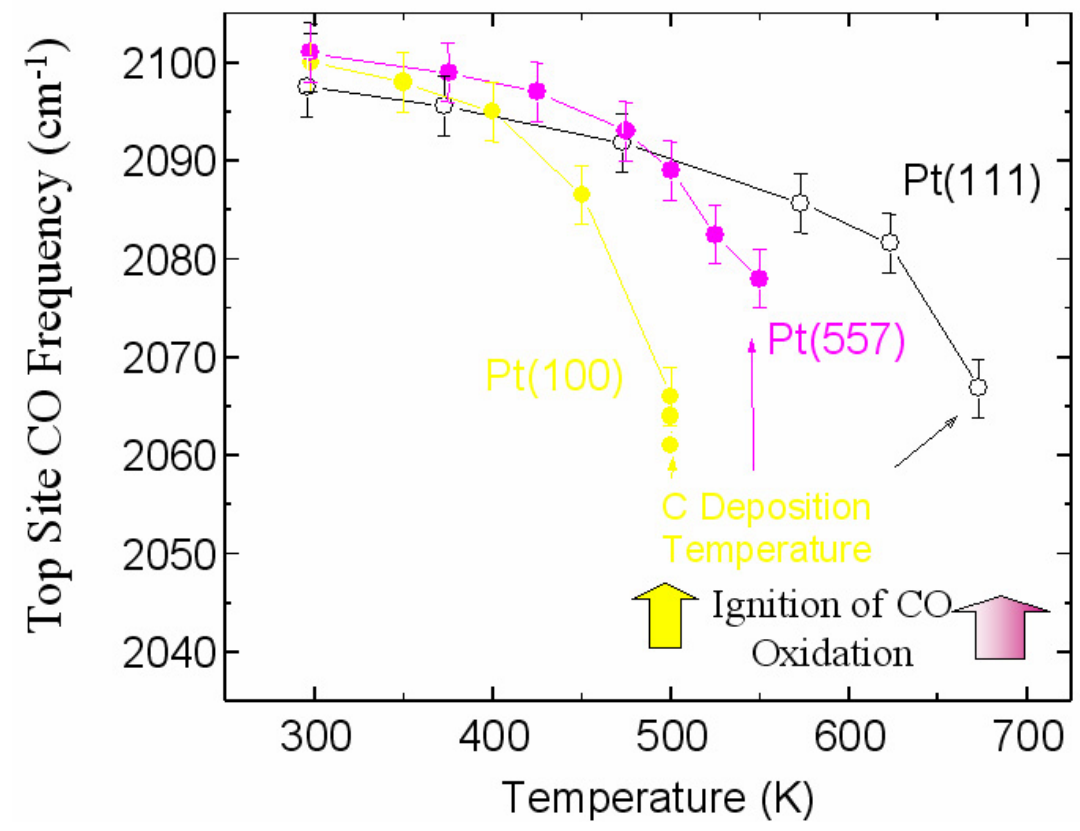

FIG. 13: The vibrational frequency of atop $\mathrm{CO}$ as a function of temperature on $\mathrm{Pt}(111), \operatorname{Pt}(100)$, and $\mathrm{Pt}(557)$.

their residence times are too long to be catalytic reaction intermediates. Thus they remain on the surface as spectators during the catalytic reactions.

Another catalytic reaction, cyclohexene hydrogenation and dehydrogenation on a $\mathrm{Pt}(111)$ surface, has been studied. SFG measurements were performed under 1.5 Torr of cyclohexene and 15 Torr of $\mathrm{H}_{2}$ at various temperatures [11]. As shown in Fig. 12, three surface intermediates are present on the surface during the reactions: 1,3-cyclohexadiene, 1,4-cyclohexadiene and cyclohexenyl. 1,3- and 1,4-Cyclohexadiene have never been observed upon cyclohexene adsorption on $\mathrm{Pt}(111)$ at low pressures $\left(<10^{-6}\right.$ Torr $)$.

The dissociation of carbon monoxide on platinum single crystal surfaces is structure sensitive [12]. As shown in Fig. 13, the (100) crystal face dissociates carbon monoxide at the lowest temperature, the stepped surface at an intermediate temperature, and the (111) face at the highest temperature. The vibrational frequency of CO shows a red shift as the temperature is increased because of a decrease in CO dipole-dipole interactions as the CO coverage decreases at higher temperature. On a given platinum surface, there is a catastrophic decline of the vibrational frequency, indicating the formation of platinum carbonyls 


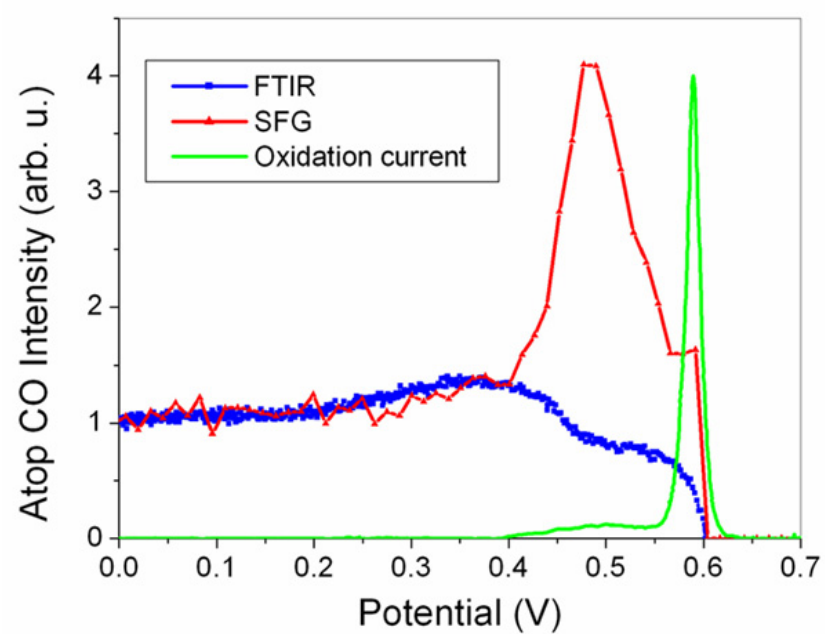

FIG. 14: The intensity of atop CO measured by FTIR (blue) and SFG (red) on the water/Pt(111) surface recorded during a positive-going potential sweep at $0.5 \mathrm{mV} / \mathrm{s}$. The green curve indicates $\mathrm{CO}$ oxidation current measured simultaneously with the spectroscopic measurements.

on the surface $\operatorname{Pt}(\mathrm{CO})_{x}$. The carbonyls then undergo a disproportionation reaction, called the Boudouard reaction, which dissociates $\mathrm{CO}$ via $2 \mathrm{CO} \rightarrow \mathrm{C}+\mathrm{CO}_{2}$. It appears that this process is very important in the ignition of $\mathrm{CO}$ oxidation on platinum surfaces. Ignition occurs when the reaction becomes suddenly so exothermic as to be self-sustaining its temperature. This happens because the deposition of carbon from the dissociation of carbon monoxide produces an additional carbon combustion reaction path, which is highly exothermic in addition to the oxidation of molecular CO.

CO oxidation can also be studied under electrochemical conditions [13]. CO pre-adsorbed on the surface can be oxidized as the applied potential increases. Figure 14 shows a comparison of CO intensity measured by Fourier transform infrared spectroscopy (FTIR) and SFG. A significant enhancement in SFG intensity was observed in the preoxidation potential region $(0.4-0.55 \mathrm{~V})$. On the other hand, the intensity measured by FTIR shows a decrease due to a decrease in number of $\mathrm{CO}$ molecules on the surface. As shown in Fig.1, SFG is a combination of IR absorption and a Raman transition. The enhancement does not come from the IR excitation. Therefore, it suggests that the enhancement comes from the Raman transition. As the intensity of Raman scattering is sensitive to the electronic states, it suggests that the electronic state of $\mathrm{CO}$ is significantly perturbed in the preoxidation region.

\section{Surface molecular structures of polymers}

Polymer surface science has two major research directions at present in our laboratory. Polymers are the steel of the 21st Century, and this may be seen in Fig. 15 in which isotactic and atactic polypropylene are shown with their elastic modulus. Just by rearranging the polymer structure, the elastic modulus can be changed by two

\section{Tacticity of Polypropylene}

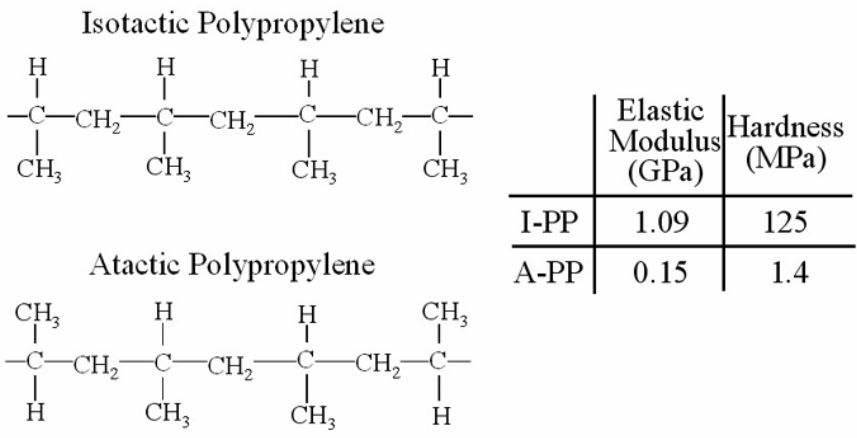

FIG. 15: The structures of isotactic and atactic polypropylene and their elastic modulus.
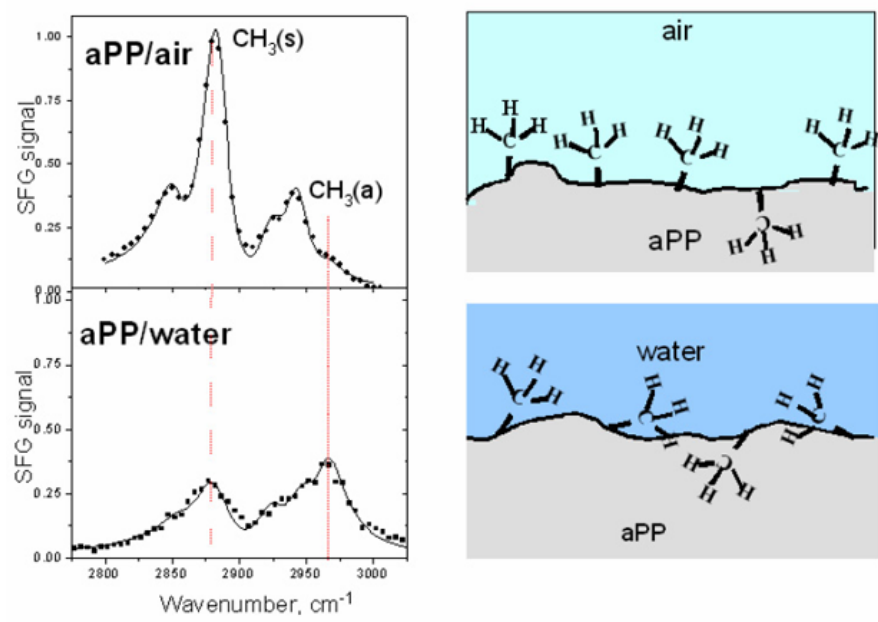

FIG. 16: The SFG spectra and proposed structures of atactic polypropylene at air and water interfaces.

orders of magnitude. The other direction of research involves biopolymer implant surfaces, such as the contact lenses or heart valves made out of polyurethane that are inserted in the human body.

Polypropylene at an air interface shows a structure where the side methyl groups in the chain are ordered, as air presents a hydrophobic interface to polypropylene. In this circumstance the methyl groups, which are considered hydrophobic, are hanging out from the polymer surface. When polypropylene is covered with water the methyl groups are disordered as shown by the SFG spectra in Fig. 16. Thus at the hydrophilic interface the methyl groups are disordered as compared to the hydrophobic polymer/air interface. When the temperature of polypropylene is altered, it undergoes a glass transition at $-10^{\circ} \mathrm{C}$, above which the polymer has lower stiffness, as shown in Fig. 17 [14]. The SFG spectral studies indicate that both the $\mathrm{CH}_{2}$ backbones and the $\mathrm{CH}_{3}$ side groups are present at the surface below the glass transition temperature and only the methyl side groups are present at the interface above the glass transition temperature. When polypropylene is embedded in methanol $\left(\mathrm{CH}_{3} \mathrm{OH}\right)$ or ace- 
(A)

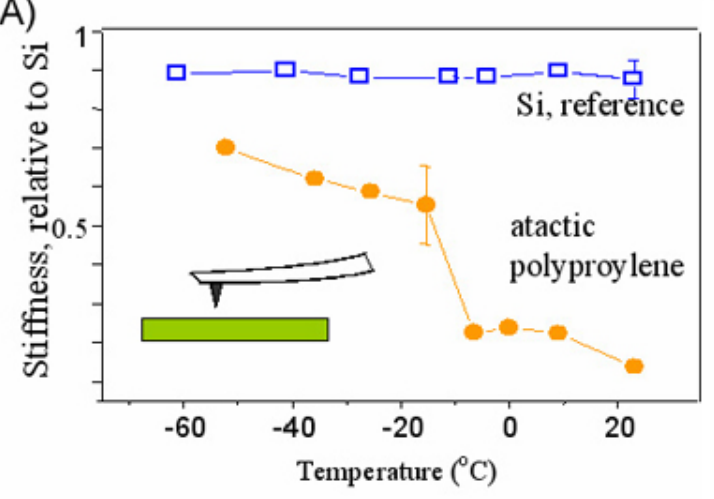

(B)

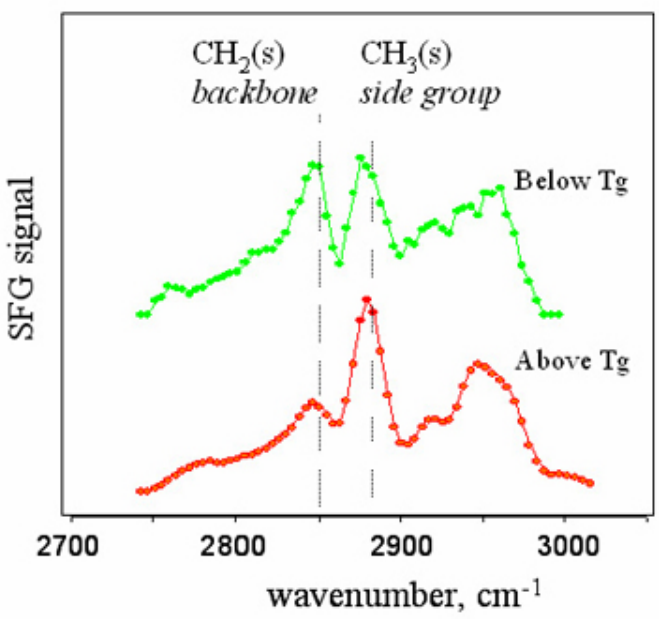

FIG. 17: (A) The relative stiffness of polypropylene measured by AFM at temperatures between -55 and $23^{\circ} \mathrm{C}$. (B) The SFG spectra of polypropylene above and below glass transition temperature.
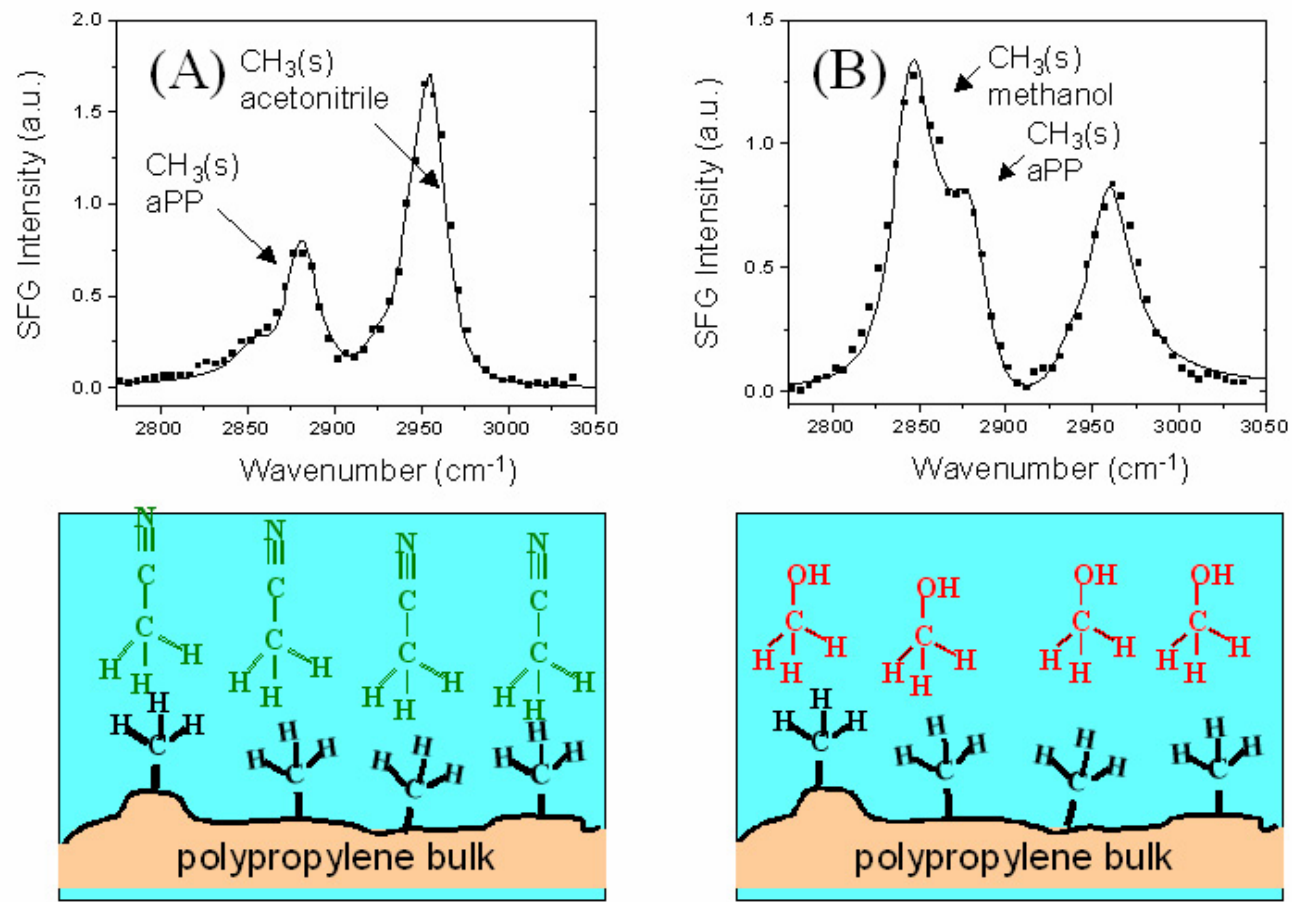

FIG. 18: The SFG spectra of polypropylene emerged in (A) methanol $\left(\mathrm{CH}_{3} \mathrm{OH}\right)$ and $(\mathrm{B})$ acetonitrile $\left(\mathrm{CH}_{3} \mathrm{CN}\right)$ solutions. The methanol and acetonitrile groups are ordered with their $\mathrm{CH}_{3}$ groups toward the surface.

tonitrile $\left(\mathrm{CH}_{3} \mathrm{CN}\right)$ solutions, the methanol and acetonitrile are also ordered with their $\mathrm{CH}_{3}$ groups toward the surface, as shown in Fig. 18.

We have shown above that hydrophobic side groups segregate to the polymer/air interface in the case of polypropylene. This is also true in the case of polystyrene. The phenyl side branches are well ordered at the air/polystyrene interface [6]. An interesting effect has been observed that involves surface segregation of end groups of polymer chains called surface modifier end (SME) groups. This is shown in Fig. 19. When polyethylene glycol has two methoxy groups at each end of the polymer chain these methoxy groups segregate to the surface and cover the surface as shown by the SFG spectra [7]. If the end groups are replaced by hydroxyl groups (OH groups), which are hydrophilic, the hydroxyl groups tuck into the bulk and do not appear on the surface. Instead the backbone of the polymer chain covers the surface. When one end of the chain is a methoxy group and the other end is $\mathrm{OH}$, the surface structure is between the two extremes. Clearly at the hydrophilic interfaces such as polymer/water interface the $\mathrm{OH}$ groups come to the surface. This is shown by a polyurethane material where the end groups were made out of silicon called poly- 

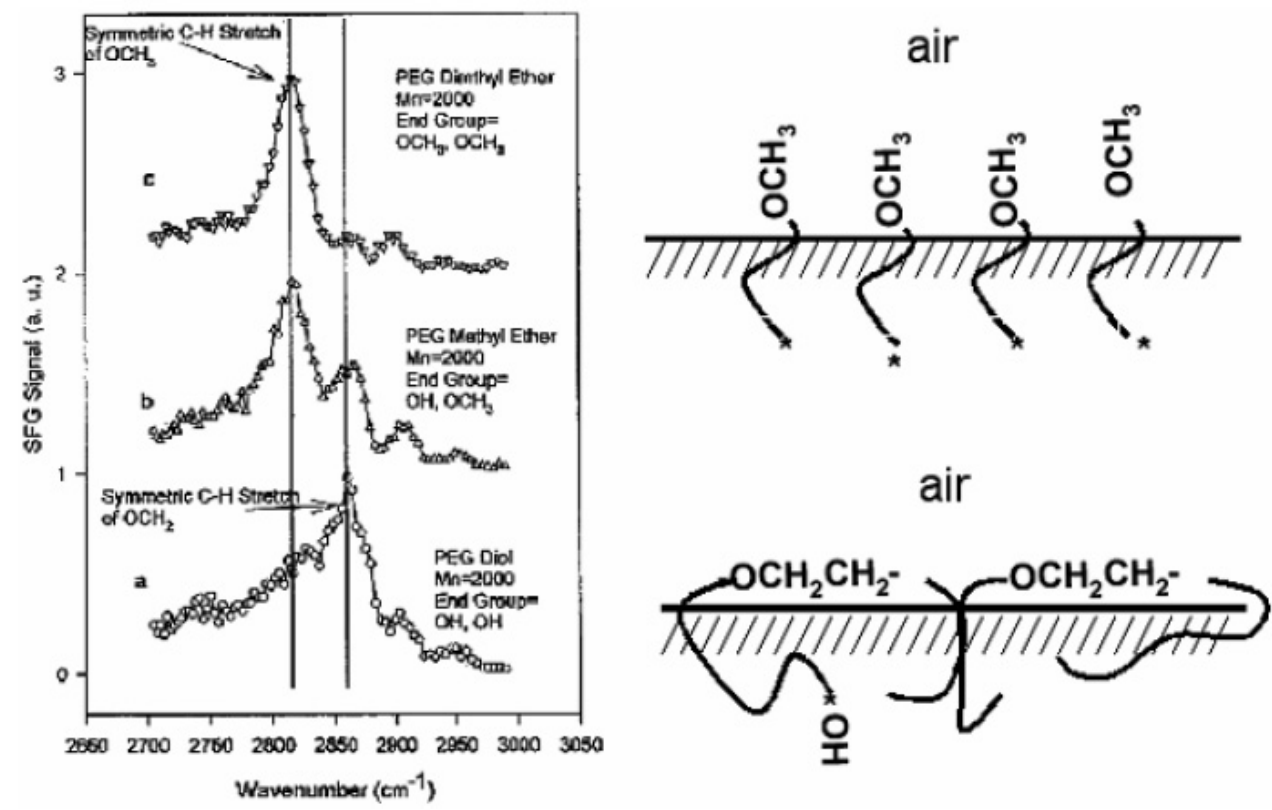

FIG. 19: The SFG spectra of poly(ethylene glycol) $\left(M_{n}=2000\right)$ with different end groups.

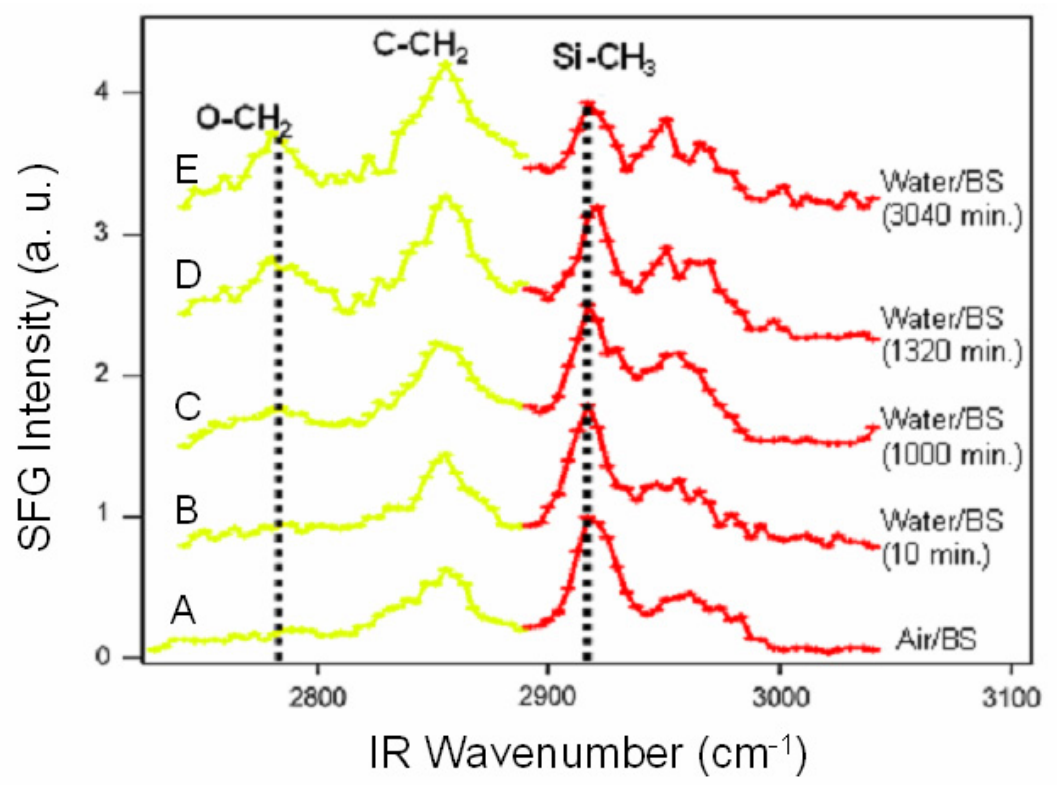

FIG. 20: (A) The SFG spectrum of air/BioSpan-S (BS) interface. (B), (C), (D), and (E) are SFG spectra of BS in water for 10, 1000, 1320, and $3040 \mathrm{~min}$, respectively.

dimethyl siloxane (PDMS) end groups [15]. Figure 20(A) shows the segregation of the silicon end groups in air to the hydrophobic interface. However, when this polymer was placed in water after two days, the silicon end groups migrate into the bulk and the more hydrophilic backbone segregates to the surface, as shown in Figs. 20(B)-(E).

In the case of polymer blends, polymers with a lower surface energy segregates to the surface. Figure 21 shows the SFG spectra of polypropylene and ethylenepropylene copolymer blends. When polypropylene and ethylene-propylene copolymer are blended, the surface is polypropylene enriched [16].
The stretching of biopolymers is another important parameter that we have to cope with in biosurface chemistry. In my laboratory there has been a major attempt to understand the changes of surface structure and surface composition as polymers stretch [17-19]. Polystyrenepolybutadiene copolymers are the common rubbers. As shown in Fig. 22, by stretching the polymer one can see a change in the surface composition. The surface changed from a primarily polystyrene covered surface, shown by the predominance of the phenyl group in the SFG spectrum, to a polybutadiene-dominated surface. Polybutadiene is the softer of the two components that cover the 


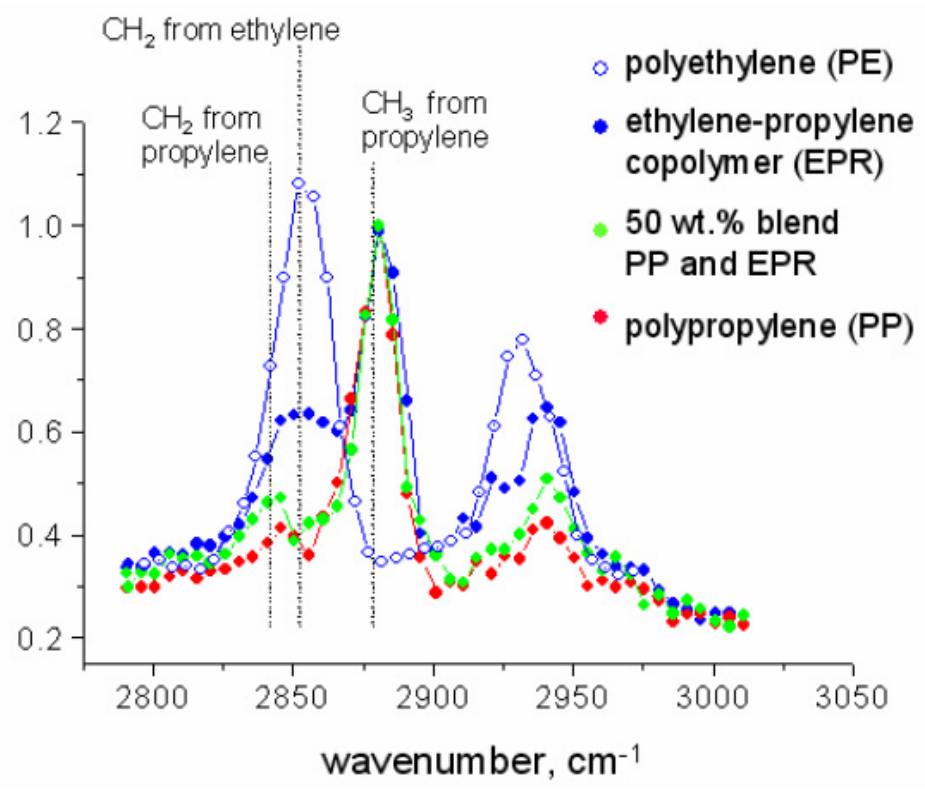

FIG. 21: The SFG spectra of polyethylene (PE), ethylene-propylene copolymer (EPR), polypropylene (PP) and ethylenepropylene copolymer blend, and polypropylene.
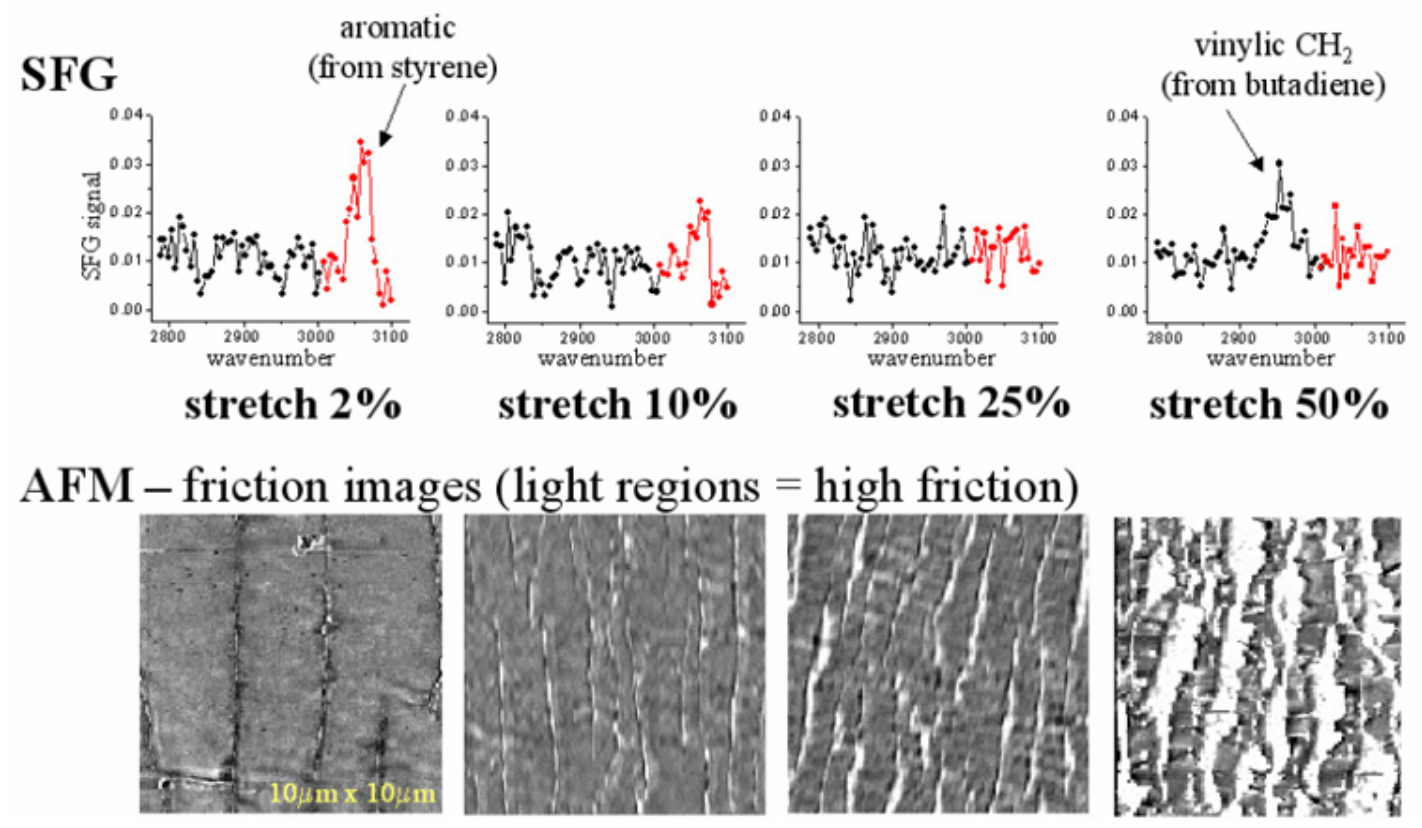

FIG. 22: The SFG spectra and AFM images of poly(styrene-butadiene-styrene) under stretch.

surface at high level of stretch. Figure 23 shows schematically the changes in the surface structure as the polymer is stretched. The anchoring rigid polymer blocks stay intact while the soft polymer components, polybutadiene in this case, is expanding as the material is stretched to cover the surface

\section{E. Protein Adsorption at Water/solid Interfaces}

Studies of proteins at polymer surfaces are essential to understand how implants respond in contact with the hu- man body. The adsorption of proteins on water/solid interfaces has been studied using SFG. Three proteins, fibrinogen, lysozyme and bovine serum albumin (BSA), were studied on hydrophilic silica surfaces and hydrophobic polystyrene surfaces [20]. Fluorescence microscopy shows that the relative amounts of protein adsorbed on hydrophilic and hydrophobic surfaces increase in proportion with the concentration of protein solutions. For a given bulk protein concentration, a larger amount of protein adsorbed on hydrophobic polystyrene surfaces compared to that on hydrophilic silica surfaces. Figure 24 shows the SFG spectra of lysozyme, fibrinogen, and BSA adsorbed 


\section{Polyethylene: a crystalline and an amorphous component}
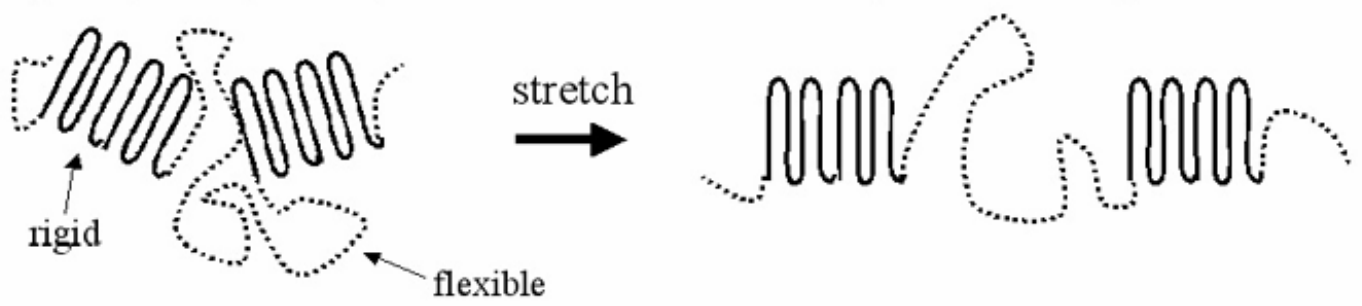

\section{Elastic block-copolymers: a glassy and a rubbery component}
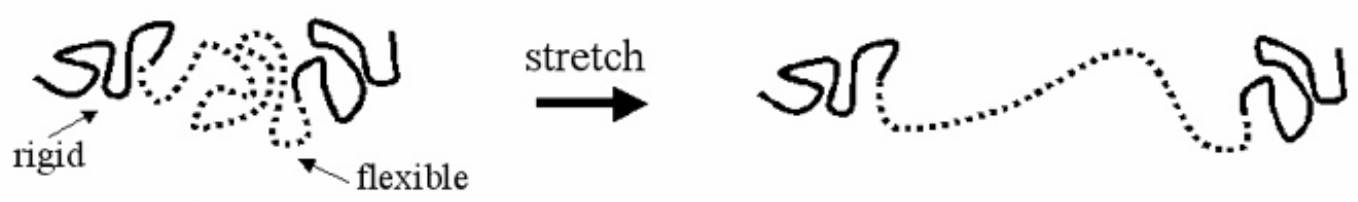

poly(styrene-butadiene-styrene)

FIG. 23: Schematics of polymers under stretch.

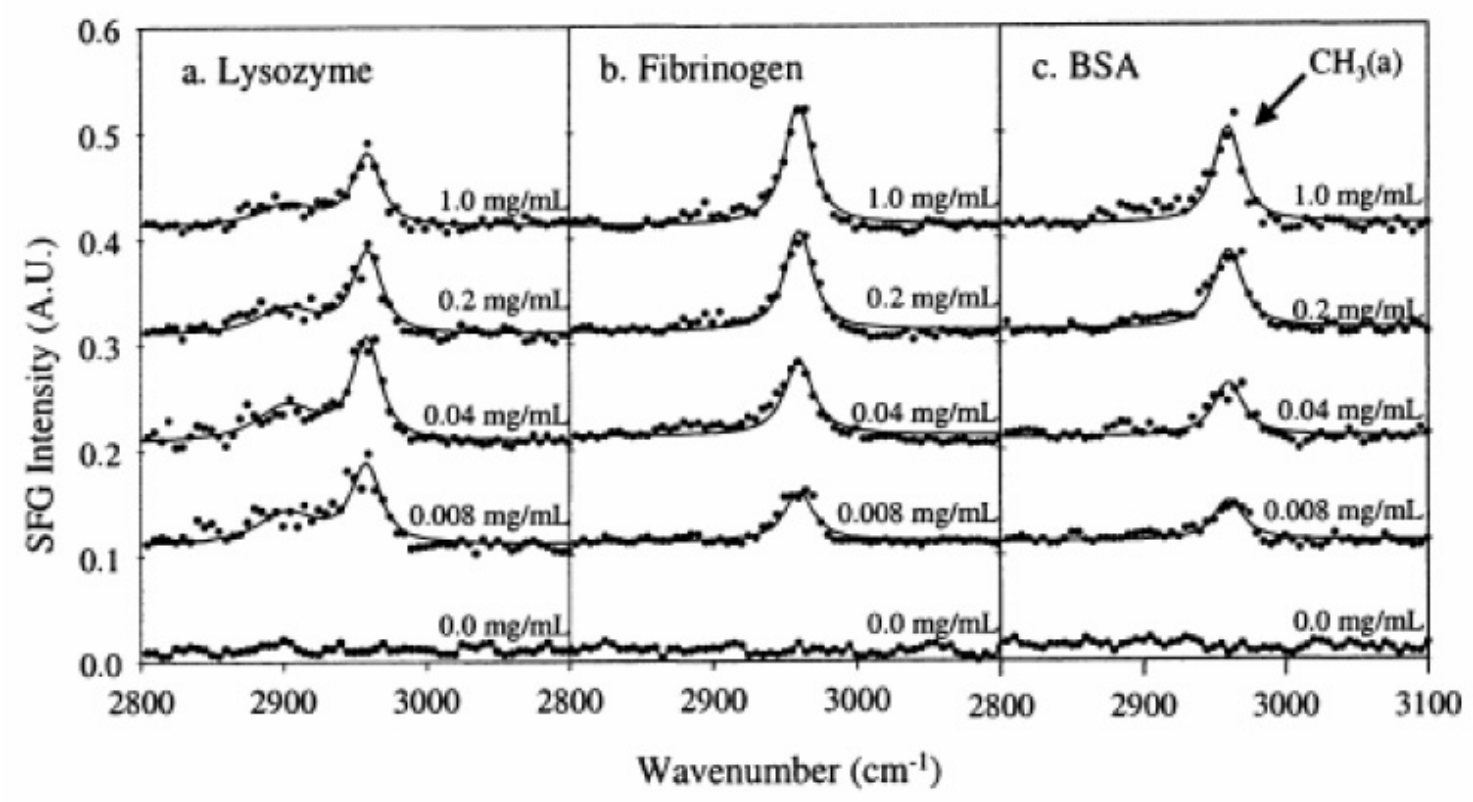

FIG. 24: SFG spectra of (a) lysozyme, (b) fibrinogen, and (c) BSA adsorbed on hydrophilic silica surfaces deposited from bulk protein solutions with concentrations of $1.0,0.2,0.04$, and $0.008 \mathrm{mg} / \mathrm{mL}$. The beam polarization combination is $\mathrm{s}$ (sum frequency), p (visible), and s (infrared).

on hydrophilic silica surfaces. Lysozyme molecules adsorbed on silica surfaces yield similar SFG spectra, regardless of the surface concentration, SFG spectra of fibrinogen and BSA on silica surfaces exhibited concentrationdependent SFG intensities and peak shapes. Quantitative SFG data analysis reveals that methyl groups in lysozyme adsorbed on hydrophilic surfaces show a concentrationindependent orientation. However, methyl groups in BSA and fibrinogen become less tilted with respect to the surface normal with increasing protein concentration at the surface, as indicated in Fig. 25. On hydropho- bic polystyrene surfaces, all proteins yield similar SFG spectra, as shown in Fig. 26. The spectra of proteins on polystyrene surfaces are different from those on hydrophilic surfaces. Although more protein molecules are present on hydrophobic surfaces, lower SFG signal intensity is observed, indicating that methyl groups in adsorbed proteins are more randomly oriented as compared to those on hydrophilic surfaces. SFG spectra also show that the orientation and ordering of phenyl rings in the polystyrene surface is affected by protein adsorption, depending on the amount and type of proteins. 

a. Lysozyme
b. Fibrinogen
c. BSA
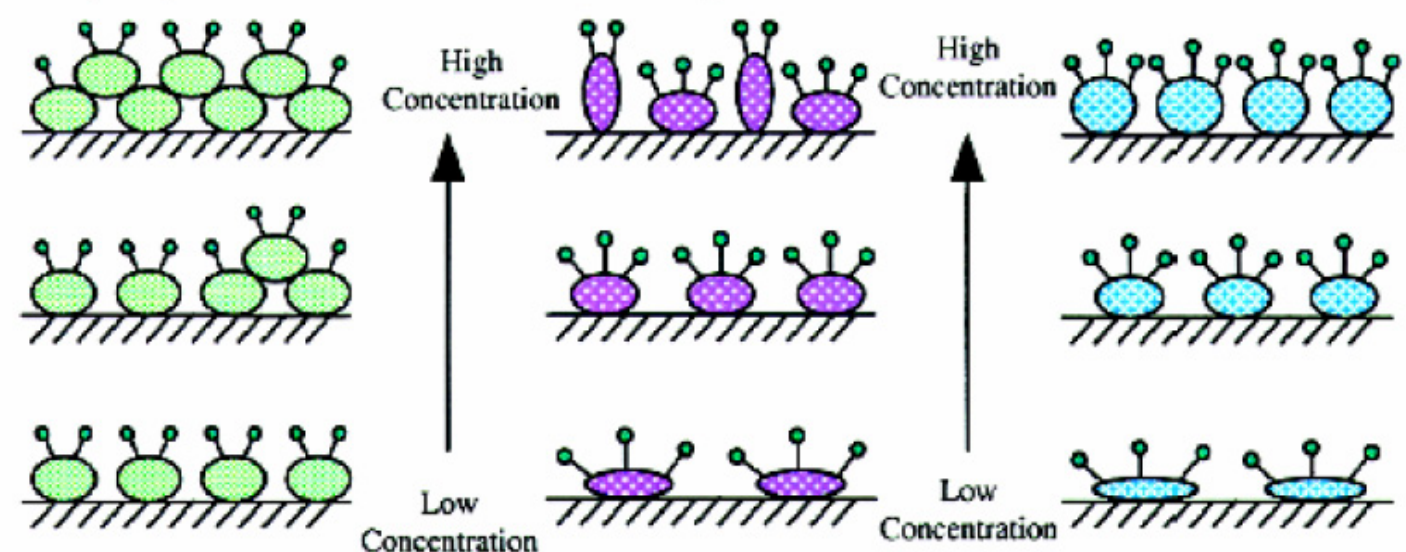

$$
\text { q = Methyl Group }
$$

FIG. 25: Proposed model for the orientation of methyl groups in (a) lysozyme, (b) fibrinogen, and (c) BSA adsorbed on hydrophilic silica surfaces with different surface concentrations.

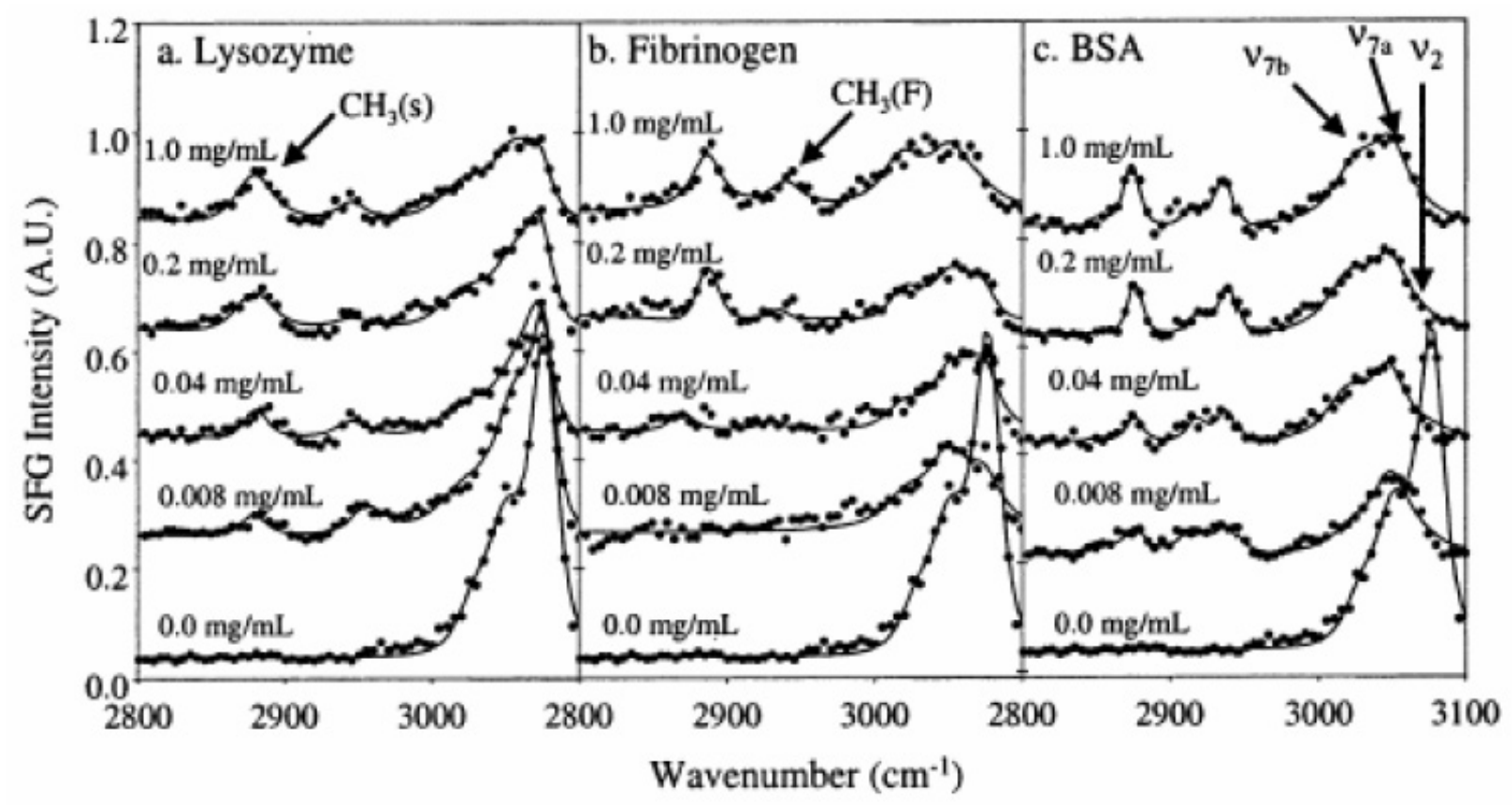

FIG. 26: SFG spectra of (a) lysozyme, (b) fibrinogen, and (c) BSA adsorbed on hydrophobic polystyrene (PS-d3) surfaces. The protein deposition is carried out in bulk solutions with concentrations of 1.0, 0.2, 0.04, 0.008, and $0.0 \mathrm{mg} / \mathrm{mL}$.

\section{CONCLUDING REMARKS}

Sum frequency generation vibrational spectroscopy opened up the buried interfaces of solid/high-pressure gas and solid/liquid to definitive molecular level studies. As a consequence we could expand our knowledge of molecular surface chemistry to these interfaces. We can also investigate more complex phenomena, such as the reaction intermediates in heterogeneous catalysis, polymer surfaces structures, and interfacial properties of proteins. SFG has been proven to be a very versatile probe for molec- ular level studies of the buried interfaces with increasing impact on surface technologies.

\section{Acknowledgments}

This work is supported by the Director, Office of Energy Research, Office of Basic Energy Sciences, Materials Sciences and Chemical Sciences Division of the U.S. Department of Energy under Contract No. DE-AC03$76 \mathrm{SF} 00098$. 
[1] P. A. Franken, A. E. Hill, C. W. Peters, and G. Weinreich, Phys. Rev. Lett. 7, 118 (1961).

[2] Y. R. Shen, Surf. Sci. 299-300, 551 (1994).

[3] Y. R. Shen, Nature 337, 519 (1989).

[4] M. Yang, D. C. Tang, and G. A. Somorjai, Rev. Sci. Instrum. 74, 4554 (2003).

[5] S. Baldelli, N. Markovic, P. Ross, Y. R. Shen, and G. Somorjai, J. Phys. Chem. B 103, 8920 (1999).

[6] A. Opdahl and G. A. Somorjai, Langmuir 18, 9409 (2002).

[7] Z. Chen, R. Ward, Y. Tian, S. Baldelli, A. Opdahl, Y. R. Shen, and G. A. Somorjai, J. Am. Chem. Soc. 122, 10615 $(2000$,$) .$

[8] S. Baldelli, G. Mailhot, P. Ross, Y. R. Shen, and G. A. Somorjai, J. Phys. Chem. B 105, 654 (2001).

[9] S. Baldelli, A. S. Eppler, E. Anderson, Y. R. Shen, and G. A. Somorjai, J. Chem. Phys. B 113, 5432 (2000).

[10] P. S. Cremer, X. Su, G. A. Somorjai, and Y. R. Shen, J. Mol. Cat. A 131, 225 (1998).

[11] M. Yang, K. C. Chou, and G. A. Somorjai, J. Phys. Chem. B 107, 5267 (2003).
[12] K. McCrea, J. S. Parker, P. L. Chen, and G. Somorjai, Surf. Sci. 494, 238 (2001).

[13] K. C. Chou, N. M. Markovic, J. Kim, P. N. Ross, and G. A. Somorjai, J. Phys. Chem. B 107, 1840 (2003).

[14] D. H. Gracias, D. Zhang, L. Lianos, W. Ibach, Y. R. Shen, and G. A. Somorjai, Chem. Phys. 245, 277 (1999).

[15] Z. Chen, R. Ward, Y. Tian, F. Malizia, D. H. Gracias, Y. R. Shen, and G. A. Somorjai, J. Biomed. Mater. Res. 62, 254 (2002).

[16] A. Opdahl, R. A. Phillips, and G. A. Somorjai, Macromolecules 35, 4387 (2002).

[17] E. Amitay-Sadovsky, K. Komvopoulos, R. Ward, and G. A. Somorjai, J. Appl. Phys. 91, 375 (2002).

[18] E. Amitay-Sadovsky, K. Komvopoulos, Y. Tian, and G. A. Somorjai, Appl. Phys. Lett. 80, 1829 (2002).

[19] E. Amitay-Sadovsky, K. Komvopoulos, R. Ward, and G. A. Somorjai, Appl. Phys. Lett. 83, 3066 (2003).

[20] J. Kim and G. A. Somorjai, J. Am. Chem. Soc. 125, 3150 (2003). 